\title{
VARIACION DEL ALMACENAJE Y ESTRATEGIAS DE RIEGO PARA ALGUNAS SERIES DE SUELO DEL DPTO. GENERAL SAN MARTIN, CHACO
}

\author{
CURRIE ${ }^{1}$, Héctor M.; SOSA ${ }^{1-2}$, Olga M.; PASAMANO², Hugo; VIRT ${ }^{1}$; Laura E. \\ ${ }^{1}$ Hidrologia Agrícola, Facultad de Ciencias Agrarias. UNNE. Sgto. Cabral 2131, TE 03783427589 - INT. 129; e-mail: \\ hmcurrie@agr.unne.edu.ar; ${ }^{2}$ Dirección de Agricultura, Ministerio de Economía, Producción y Empleo, \\ Gobierno del Chaco. M.T de Alvear 145; 7 mo Piso,Resistencia, Chaco.
}

\begin{abstract}
RESUMEN
Los suelos retienen humedad de acuerdo a características físicas, tales como textura y estructura. El agua aprovechable que puede contener un suelo está directamente relacionada con su textura. A fin de elaborar estrategias de riego para una región dada deben ser establecidas para cada caso con sus particularidades, se efectuó la evaluación de la variación del almacenaje de agua en diferentes series de suelos del Dpto. General San Martín, Provincia del Chaco. Para ello se realizó en campaña ensayos de infiltración con anillos concéntricos, cuyos resultados fueron evaluados con la ecuación de Kostiakov. Fueron muestreadas 10 series de suelos, para la posterior determinación de densidad aparente por método de la probeta y humedad equivalente y punto de marchites permanente por método gravimétrico. Mediante el uso de GPS se relevaron las coordenadas geográficas de los lugares de ensayo, para la ubicación en los mapas de suelo. El contenido de humedad en capacidad de campo, punto de marchites permanente y la capacidad de almacenamiento de agua del suelo difieren para cada serie de suelo así como la tasa de infiltración básica y la lámina bruta. Atendiendo la variabilidad que se manifiesta en las condiciones físicas de los suelos en una región dada no puede establecerse "a priori" de forma generalizada la lámina de riego, la frecuencia ni la duración de los riegos a aplicar. En una región dada para poder establecer una adecuada estrategia de riego, es necesario contar con datos de la física del suelo donde va a realizarse el riego.
\end{abstract}

Palabras clave: irrigación, capacidad de campo, almacenamiento.

\begin{abstract}
Each type of soil can contain humidity according to their physical characteristics, such as texture and structure. In order to implement irrigation technology it is necessary to know the humidity present in the soil, which is then used to calculate the foil of water consumed in combination with other parameters, or even to define when to irrigate. The amount of available water that can be held in a soil (or: "that a soil can hold") is directly related to its texture. In order to elaborate and demonstrate that irrigation strategies for a given region, should be established for each case with their attributes, it was carried on an evaluation of variations on water storage for different soil series of the General San Martín Departament, in Chaco Province. In this way it was conducted an infiltration test with concentrics rings, whose results were evaluated with the Kostiakov equation. 10 soil series were sampled for the subsecuent determination of apparent density by the graduated test tube method, and equivalent humidity and permanent wither point by gravimetric method. Also, a GPS was used to get test site geographic coordinates, for their location on soil maps. The humidity content in field capacity, in permanent withers point and the water storage capacity of soils differ for each series of soil as well as the rate of infiltration and gross foil. In view of the variability observed in physical conditions of soils, it should not be establish a priori or in a general way neither the irrigation foil, nor the frecuency and duration of irrigation to be applied.
\end{abstract}

Key words: irrigation, equivalent humidity, storage capacity

\section{INTRODUCCION Y ANTECEDENTES}

El fin del riego es aportar agua para obtener una cosecha óptima en cantidad y calidad. El cálculo y programación de riego en la parcela se debe determinar, de la manera más exacta posible cuándo hay que regar, cuánto hay que regar y cuánto tiempo se tarda en la aplicación de dicho riego (Castañón, 2000).
Para realizar una programación del riego es necesario conocer la cantidad de agua existente en el suelo. En general, dicho dato es difícil de obtener, se suele dar un riego a comienzo de la campaña, con el que el terreno estará prácticamente a capacidad de campo y la reserva de agua será máxima. A dicha reserva se van sumando los aportes (riegos, lluvias) 
y restando los consumos (evapotranspiración), por períodos de tiempos más o menos largos. Cuando la reserva alcance un valor preestablecido, por debajo del cual puede sufrir el cultivo, es necesario regar (Castañón, 2000.).

La dosis de agua que se aplica en cada riego por unidad de superficie depende de las condiciones hidrofísicas del terreno y de la profundidad de las raíces del cultivo (Castañón, 2000)

Las constantes hídricas del suelo, a saber, capacidad de campo (CC), punto de marchites permanente (PMP), capacidad de almacenamiento o humedad aprovechable (HA); están relacionadas a la textura, la estructura, el tamaño y distribución de poros, la temperatura y a la histéresis. Estas constantes hídricas son expresadas como un porcentaje respecto del suelo seco.

El suelo almacena el agua y la pone a disposición de las plantas dependiendo del contenido de humedad y potenciales de agua.

Las condiciones ideales de abastecimiento de agua difieren entre los distintos cultivos, cuando estas condiciones no son proporcionadas la planta comienza a sufrir "stress hídrico".

El umbral hídrico representa el mínimo contenido de humedad para no limitar el crecimiento y generalmente se expresa como una tensión o succión. También puede expresarse la humedad óptima como porcentaje de agua útil.

La retención de humedad es la capacidad de los suelos de almacenar agua y varía conforme a las propiedades que le confieren la textura, los coloides, tamaño y distribución de poros, contenido de materia orgánica, entre otros factores. Esta retención de humedad está íntimamente ligada a la disponibilidad de agua para las plantas.

Durante períodos relativamente cortos dicha capacidad de almacenamiento de agua puede mantenerse constante, sin embargo el manejo de suelos puede modificarla en sentido positivo o negativo (p.e. adición de materia orgánica, compactación respectivamente).

Es necesario conocer el contenido de humedad del suelo para realizar el cálculo de la lámina de riego consumida, en combinación con otros parámetros, o bien para definir cuando regar.

La cantidad de agua que se necesita para saturar una determinada área de suelo seco, depende de sus características texturales y estructurales, así como la profundidad hasta la que se quiere humedecer (Palacio Vélez, 1994).

Conforme el suelo se va secando, la diferencia de potencial o presión entre el agua en el suelo y en la raíz disminuye progresivamente, hasta que en un momento dado, esta diferencia es tan pequeña que la velocidad de entrada de agua en la raíz es menor que la velocidad de salida por las hojas, iniciándose el proceso de marchitamiento y si el proceso continúa la planta puede morir, en cuyo caso se dice que el suelo alcanzó el porcentaje de humedad de marchitamiento permanente o punto de marchites permanente (PMP) (Palacios Vélez, 1994.).

También en este caso el contenido de humedad y el potencial con que el agua está retenida, es variable y depende no sólo del suelo sino también de la planta (Palacios Vélez, 1994.).

Según Palacios Vélez (1994) no puede permitirse que los cultivos consuman toda el agua aprovechable que puede almacenar el suelo. En la primer etapa de desarrollo de los cultivos, las plantas pueden resistir mayores tensiones de humedad, sin embargo a partir de la floración deberá ser menor, para lograr buenos rendimientos, por esta razón no es recomendable utilizar en ésta etapa más del 50\% o $60 \%$ de la lámina útil, y en las etapas iniciales hasta un $70 \%$ de la lámina aprovechable, lo cual también depende del cultivo.

La Infiltración se define como el proceso por el cual el agua penetra por la superficie del suelo y llega hasta sus capas inferiores (Velez et al., 2002; citado por Ruiz Estévez et al.,2004).

La determinación de la velocidad de infiltración es una de las prácticas más importantes a desarrollar en la práctica de sistematización de tierras a regar.

Si se aplica agua a un área del terreno, la lámina infiltrada disminuye con respecto al tiempo, evidenciando una disminución en la velocidad de infiltración (Chambouleyron, 2005).

Mientras que la velocidad de aporte de agua a la superficie del suelo es menor que la infiltrabilidad, el agua se infiltra tan rápidamente como es aportada, lo cual manifiesta que la velocidad de aporte determina la velocidad de infiltración. Sin embargo, existe también la posibilidad que la velocidad de aporte exceda la infiltrabilidad del suelo y en ese mismo momento ésta última es la que determina la velocidad real de infiltración; de ese modo el proceso es controlado por las características del perfil (Gurovich, 1985; citado por Ruiz Estévez, 2004).

Los valores de velocidad de infiltración que se obtienen en el campo, presentan una gran variabilidad en cuanto a su distribución espacial, debido a la influencia de: (a) características del suelo; (b) características del perfil del suelo; (c) niveles de humedad del suelo; (d) método de riego y manejo del agua; (e) otros factores (influencia de la temperatura, aire atrapado, etc.) (Chambouleyron, 2005.).

En Tabla $N^{0} 1$ se caracterizan los suelos según la infiltración básica; esta escala resulta de promediar lo propuesto por Philips (1957), quien asocia la misma a familias de suelos, y la interpretación que formula Kostiakov (1932) 
Tabla $\mathbf{N}^{\mathbf{0}}$ 1: Familias de infiltración. Procedimiento del Servicio de Conservación de Suelos (Philips, 1957) y de Kostiakov(1932).

\begin{tabular}{lc}
\hline Característica del suelo & $\mathbf{l b}(\mathbf{m m} / \mathbf{h})$ \\
\hline Muy baja & 2,5 \\
Baja & 7,5 \\
Moderada baja & 12,5 \\
Moderada & 25,0 \\
Moderada rápida & 37,0 \\
Rápida & 50,0 \\
Muy rápida & 75,0 \\
Extremadamente rápida & 100 \\
\hline
\end{tabular}

\section{OBJETIVOS}

Establecer el almacenaje hídrico para algunas series de suelos con antecedentes agrícolas del Departamento San Martín, Chaco,

Proponer estrategias de riego a partir de variables tales como la infiltración y el almacenaje en el suelo.

\section{MATERIALES Y METODOS}

Se ha realizado el muestreo de los suelos, que corresponden a las series que poseen antecedentes de actividad agrícola. Los muestreos realizados fueron tres (loma-media loma y bajo), en función de la profundidad del horizonte superficial, la misma está expresada en la Tabla 2 ( Ledesma y Zurita, 1988), en la misma además, se vuelca información relativa a cada una de las series con antecedentes agrícolas.

Para la determinación de humedad equivalente se utilizó el método gravimétrico y por el método de la probeta, se obtuvo la densidad aparente (Bouyucos, 1963).

También se efectuaron ensayos de infiltración con anillos concéntricos (Método de Muntz) (Burt, 1995), cuyos resultados fueron analizados con la ecuación de Kostiakov.

Con el relevamiento de coordenadas geográficas tomadas con GPS E-Trex Legend, y el uso de los software Mapsource ${ }^{\circledR}$ y Google Earth ${ }^{\circledR}$, se determinó la ubicación de los lotes muestreados en el Plano Catastral, para su posterior traspolación a la Carta de Suelos del Departamento General San Martin, Chaco. (Ledesma y Zurita, 1988).

A partir de la ubicación de los lotes en el mapa de suelo se tomaron de las muestras de las series de suelo que corresponden a cada lote.

\section{RESULTADOS}

Los resultados de los análisis del laboratorio y de infiltración básica que se hallan expuestos en el Tabla $\mathrm{N}^{\circ} 3$, manifiestan claramente que los factores en estudio difieren en las series de suelo anteriormente mencionadas. En la Tabla $n^{\circ} 3$ y para cada una de las series identificadas se consigna la capacidad de campo (CC \%), el punto de Marchitez permanente (PMP $\%$ ), agua disponible (HA,\%), densidad aparente $(\delta \mathrm{a}$, $\mathrm{kg} \mathrm{dm}^{-3}$ ), e infiltración básica $\left(\mathrm{Ib}, \mathrm{mm} \mathrm{hora}^{-1}\right)$; clasi-

Tabla $N^{0}$ 2: Series de suelo muestreadas del Departamento General San Martín.

\begin{tabular}{|c|c|c|c|c|c|c|c|c|c|c|c|}
\hline \multicolumn{2}{|c|}{ Serie de Suelo } & $\begin{array}{l}\overline{\frac{\pi}{0}} \\
\text { 듕 }\end{array}$ & $\begin{array}{l}\text { एँ } \\
\text { ثैँ }\end{array}$ & 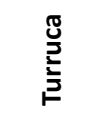 & $\begin{array}{l}\frac{\overline{5}}{5} \\
\frac{5}{4}\end{array}$ & $\frac{\circ}{\frac{0}{\bar{\sigma}}}$ & 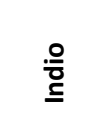 & $\begin{array}{l}\frac{\pi}{0} \\
\frac{\pi}{5} \\
5 \\
5\end{array}$ & 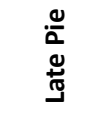 & ț & 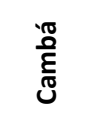 \\
\hline \multicolumn{2}{|c|}{ Profundidad (cm) } & $0-14$ & $0-20$ & $6-21$ & $0-9$ & $0-10$ & $0-12$ & $0-14$ & $0-15$ & $0-12$ & $0-4$ \\
\hline \multicolumn{2}{|c|}{ Factor de humedad } & 1,02 & 1,02 & 1,03 & 1,03 & 1,04 & 1,04 & 1,06 & 1,05 & 1,04 & 1,01 \\
\hline \multirow{3}{*}{ 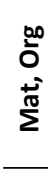 } & C (\%) & 1,22 & 1,31 & 0,83 & 2,00 & 2,95 & 2,92 & 4,05 & 3,38 & 1,07 & 0,35 \\
\hline & $\mathrm{N}(\%)$ & 0,109 & 7 & 0,144 & 0,230 & 0,359 & 0,268 & 0,501 & 0,418 & 0,117 & 0,035 \\
\hline & $\mathrm{C} / \mathrm{N}$ & 11 & 27,1 & 6 & 9 & 8 & 10 & 8 & 8 & 9 & 10 \\
\hline \multirow{9}{*}{ 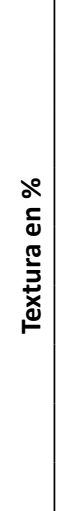 } & Arcilla $(<2 \mu)$ & 20,1 & 31,7 & 15,2 & $15,6 x$ & 22,2 & 29,7 & 49,4 & 39,8 & 19,5 & 2,5 \\
\hline & Limo $(2-20 \mu)$ & 29,3 & 69 & 35,4 & 25,6 & 28,2 & 27,7 & 29,2 & 29,4 & 40,4 & 18,4 \\
\hline & Limo $(2-50 \mu)$ & 73,2 & 3,4 & 83,2 & 77,4 & 69,8 & 63,6 & 47,7 & 50,2 & 65,9 & 43,7 \\
\hline & Arena $\mathrm{m}$, fina $1(50-74 \mu)$ & 6,1 & 0,3 & 0,8 & 3,8 & 5,8 & 5,6 & 2,0 & 7,9 & 6,6 & 13,2 \\
\hline & Arena $\mathrm{m}$, fina $2(74-100 \mu)$ & 0,6 & 0,2 & 0,0 & 1,8 & 1,5 & 0,3 & 0,3 & 0,7 & 1,4 & 15,3 \\
\hline & Arena fina $(100-250 \mu)$ & 0,0 & 0,2 & 0,4 & 1,3 & 0,5 & 0,4 & 0,4 & 1,4 & 3,7 & 25,2 \\
\hline & Arena media $(250-500 \mu)$ & & 0 & 0,4 & 0,1 & 0,2 & 0,4 & 0,2 & 0 & 0,1 & 0,1 \\
\hline & Arena gruesa $(500-1000 \mu)$ & 6,7 & & & & & & & & 2,8 & \\
\hline & Arena $\mathrm{m}$, gruesa $(1000-2000 \mu)$ & & 3,9 & 1,6 & 7,0 & 8,0 & 6,7 & 2,9 & 10 & & 53,8 \\
\hline
\end{tabular}


Tabla No 3: Resultados de los análisis de suelo y cálculos de agua disponible, lámina de agua disponible, volumen de agua disponible y lámina bruta de las series de suelo.

\begin{tabular}{|c|c|c|c|c|c|c|c|}
\hline SERIE & $\begin{array}{l}\mathrm{CC} \\
(\%)\end{array}$ & $\begin{array}{l}\text { PMP } \\
(\%)\end{array}$ & $\begin{array}{l}\text { HA } \\
(\%)\end{array}$ & $\begin{array}{c}\delta \mathrm{a} \\
\left(\mathrm{kg} \mathrm{dm}^{-3}\right)\end{array}$ & $\begin{array}{c}\mathrm{lb} \\
\left(\mathrm{mm} \text { hora }^{-1}\right)\end{array}$ & $\begin{array}{l}\text { Característica del suelo } \\
\text { en función de la I b }\end{array}$ & $\begin{array}{l}\text { Lamina bruta } \\
\left(\mathrm{mm} \mathrm{ha}^{-1}\right)\end{array}$ \\
\hline CABRAL & 23,83 & 12,98 & 10,84 & 1,08 & 25,09 & Moderada & 11,75 \\
\hline COTTERA & 35,17 & 19,11 & 16,06 & 1,10 & 65,24 & Rápida & 17,67 \\
\hline TURRUCA & 17,86 & 9,82 & 8,04 & 1,25 & 0,52 & Muy baja & 10,05 \\
\hline AUSTRAL & 15,54 & 8,55 & 6,99 & 1,20 & 2,99 & Muy baja & 8,39 \\
\hline MENJO & 13,39 & 7,28 & 6,11 & 1,20 & 3,17 & Muy baja & 7,33 \\
\hline INDIO & 24,87 & 13,52 & 11,35 & 1,15 & 3,81 & Muy baja & 13,05 \\
\hline UNIDAS & 30,36 & 16,5 & 13,86 & 1,12 & 0,20 & Muy baja & 15,52 \\
\hline LATEPIE & 20,97 & 11,4 & 9,57 & 1,08 & 25,07 & Moderada & 10,34 \\
\hline WINTER & 19,77 & 10,74 & 9,03 & 1,120 & 47,11 & Moderada rápida & 10,11 \\
\hline CAMBA & 15,76 & 8,57 & 7,19 & 1,11 & 2,42 & Muy baja & 7,98 \\
\hline MEDIOS & 22,97 & 12,51 & 9,30 & 1,15 & 21,89 & Moderada baja & 11,81 \\
\hline MAXIMOS & 35,17 & 19,11 & 16,06 & 1,25 & 65,24 & Rápida & 17,67 \\
\hline MÍNIMOS & 13,39 & 7,28 & 6,11 & 1,08 & 0,197 & Muy baja & 7,33 \\
\hline
\end{tabular}

ficación del suelo en función de la infiltración básica (Ib), para finalmente obtener la lámina bruta ( $\mathrm{Lb} \mathrm{mm}$ $\mathrm{ha}^{-1}$ ), para cada una de las series estudiadas

\section{DISCUSION}

En los resultados obtenidos de la evaluación de las diez series de suelos estudiadas, se observan que el contenido de humedad para CC, PMP, HA, Ib y Lámina Bruta difieren para cada serie de suelo. En tal sentido y siguiendo a Castañon (2000), es posible inferir que la estrategia de riego, en términos de volúmenes y dosis difieren para cada uno de los suelos analizados.

Por otra parte, y coincidiendo con Palacio Velez (1994) la baja Humedad Disponible (HA\%), que en el mejor de los casos solo alcanza hasta el $16.06 \%$, implica una estrategia de riegos frecuentes, y por tiempos relativamente cortos, debido a los bajos valores de Infiltración Básica, en cuyo caso algunos suelos, solo alcanzan a $0.2 \mathrm{~mm} \mathrm{hora}^{-1}$. Esta puede ser altamente variable (Chambouleyron, 2005), y lo es en los suelos analizados, que por ej. en la serie Cottera alcanza a $65.24 \mathrm{~mm}$ hora; mientras que en la serie Unidas es de $0.2 \mathrm{~mm}$ hora $^{-1}$.

Analizando HA, se destaca la serie de suelo Cottera presentando el máximo valor para éste parámetro con $16,06 \%$, seguida por las series Unidas, Indio y Cabral con $13,86 \%, 11,35 \%$ y $10,84 \%$ respectivamente. Esto significa que son los suelos con mayor capacidad de almacenar agua en forma aprovechable para los cultivos.
Las series de suelo con los valores inferiores de HA resultaron ser Menjo, Austral y Cambá con $6,11 \%, 6,99 \%$ y $7,19 \%$ respectivamente.

Falasca et al. (2002) en un trabajo de Simulación de las necesidades de riego complementario de un sector del partido de Tandil, República Argentina, analizando las series de suelos del área de estudio, hallaron que la serie Mar del Plata se correspondía con una menor necesidad de riego por tratarse de la serie con mayor capacidad de almacenaje de agua y la mayor necesidad de riego se observó en los suelos de la serie Balcarce.

Análogamente, luego de una lluvia profusa o riego, considerando en condiciones climáticas similares y de cultivo, es probable que la necesidad de riego se presente antes en las series de suelo Menjo, Austral o Cambá con respecto a las series de suelo Cottera, Unidas o Indio, dado que pueden almacenar menor cantidad de agua, la cual va a ser consumida antes por el cultivo.

La tasa de la Ib representa la velocidad con la que ingresa el agua en el suelo, y se relaciona por lo tanto el tiempo de riego en función de aquella. De las series de suelo estudiadas, resultó la serie Cottera la de mayor Ib y la serie Unidas la de menor Ib.

Entonces, para que la velocidad de infiltración sea controlada por la velocidad de aporte del agua, este caudal debe ser determinado para cada serie de suelo en función de su Ib. Si la velocidad de aporte de agua a la superficie del suelo supera la velocidad de la infiltración, el agua se perderá por escurrimiento en caso de suelos con pendiente, o quedará encharcada, en si el terreno es más plano. 
La lámina bruta representa la cantidad de agua (expresado en $\mathrm{mm} \mathrm{ha}^{-1}$ ) que puede contener el suelo en forma disponible para la planta.

En éste estudio se han calculado las láminas brutas para $10 \mathrm{~cm}$ de profundidad efectiva de raíces por tratarse de suelos empleados para cultivos hortícolas.

Tomando como ejemplo de la Tabla No 2 la Serie de Suelo Menjo, cuya lámina bruta es de 7,33 $\mathrm{mm} \mathrm{ha}^{-1}$, un aporte mayor a la lámina bruta se traducirá no tendrá influencia positiva en el aporte de riego. Esta lámina debe aportarse a una velocidad no superior $3,17 \mathrm{~mm} \mathrm{ha}^{-1}$, por tanto el tiempo aproximado de riego será de 2 horas 18 minutos.

El intervalo de tiempo que transcurre entre dos riegos consecutivos depende de la evapotranspiración. Sin embargo, como línea general, la lámina de reposición debe aplicarse cuando se haya consumido entre el 50 y el $70 \%$ de la $\mathrm{Lb}$, con un volumen tal que no supere a dicha $\mathrm{Lb}$.

\section{CONCLUSIONES}

Atendiendo la variabilidad que se manifiesta en las condiciones físicas de los suelos en una región dada no debe establecerse a priori ni en forma generalizada la lámina de riego, la frecuencia ni la duración de los riegos a aplicar.

De modo entonces que la estrategia de riego debe estar sustentada en el comportamiento local de los atributos físicos del suelo que regulan la capacidad de almacenamiento, la profundidad del suelo y la infiltración básica.

\section{BIBLIOGRAFIA}

Bouyoucos, G. J. 1963. Direction for making mechanical analysis of soil by hydrometer methods. Soil Sci. 42: $25-30$.
Burt, CM. 1995. The Surface Irrigation Manual - A Comprehensive Guide to Design and Operation of Surface Irrigation Systems. Waterman Industries. Exeter, CA. 373 p.

Castañón, G. 2000. Ingeniería del Riego. Utilización Racional del Agua. International Thomson Editores Spain. Paraninfo S.A., Madrid, España.

Chambouleyron, J. 2005. Riego y Drenaje. Técnicas para el desarrollo de una agricultura regadía sustentable. Tomo I. EDIUNC, Mendoza, Argentina.

Falasca, S.L.; Forte Lay, J.A.; Bernabé, M.A. 2002 Simulación De Las Necesidades De Riego Complementario De Un Sector Del Partido De Tandil, República Argentina. Revista Brasileira de Agrometeorelogía, Santa Maria, V 10 N²: 333-342.

Kostiakov, A. N. 1932. On the dynamics of the coefficients of water percolation in soils and on the necessity of studying it from a dynamic point of view for purpose of amelioration. Transactions of the 6th Communication of the Int. Society of Soil Sciences, Part A: 17-21.

Ledesma L. L. y Zurita J. J. 1988. Cartas de Suelos de la República Argentina - Provincia del Chaco -Los Suelos del Departamento Gral. San Martín.

Palacio Velez, E. 1994.Técnicas para la evaluación y mejoramiento de la operación de los distritos de riego. Tesis de Maestría, Colegio de Postgraduados. Chapingo, México.

Philip, J. R. 1957. The theory of infiltration: 5. The influence of the initial moisture content. Soil Sci., 84: 329-339.

Ruiz Estévez, F.; Venialgo, C.A.; Gutierrez, N.C.; Ingaramo, O.; Briend, M.C. 2004. Infiltración De Agua En El Suelo Con Diferentes Usos En El Departamento 9 De Julio (Chaco). Universidad Nacional Del Nordeste, Comunicaciones Científicas y Tecnológicas. 